\title{
ADAPTIVE FINITE ELEMENT METHODS FOR MIXED CONTROL-STATE CONSTRAINED OPTIMAL CONTROL PROBLEMS FOR ELLIPTIC BOUNDARY VALUE PROBLEMS
}

\author{
R.H.W. HOPPE AND M. KIEWEG
}

\begin{abstract}
Mixed control-state constraints are used as a relaxation of originally state constrained optimal control problems for partial differential equations to avoid the intrinsic difficulties arising from measure-valued multipliers in the case of pure state constraints. In particular, numerical solution techniques known from the pure control constrained case such as active set strategies and interior-point methods can be used in an appropriately modified way. However, the residual-type a posteriori error estimators developed for the pure control constrained case can not be applied directly. It is the essence of this paper to show that instead one has to resort to that type of estimators known from the pure state constrained case. Up to data oscillations and consistency error terms, they provide efficient and reliable estimates for the discretization errors in the state, a regularized adjoint state, and the control. A documentation of numerical results is given to illustrate the performance of the estimators.
\end{abstract}

Keywords: distributed optimal control problems, mixed control-state constraints, adaptive finite elements, a posteriori error analysis,

AMS/MOS Classification: 65N30, 65N50; 49K20, 65K10

\section{INTRODUCTION}

Adaptive finite element methods based on reliable a posteriori error estimators are powerful algorithmic tools for the efficient numerical solution of boundary and initial-boundary value problems for partial differential equations (PDEs) (cf. [1, 3, 4, 14, 35, 41] and the references therein). On the other hand, considerably less work has been done with regard to optimal control problems for PDEs. The so-called goal oriented dual weighted approach has been applied in the unconstrained case in $[4,5]$ and to control constrained problems in $[20,42]$, whereas

Date: April 9, 2007. 
residual-type a posteriori error estimators for control constrained problems have been derived and analyzed in [16, 17, 21, 25, 28, 30, 31]. Unlike the control constrained case, pointwise state constrained optimal control problems are much more difficult to handle due to the fact that the Lagrange multiplier for the state constraints lives in a measure space (see, e.g., [8, 9, 23, 39]). Therefore, it is a natural idea to regularize state constrained problems by means of mixed control-state constrained ones, since with regard to numerical solution techniques the regularized problems can be formally treated as in the case of pure control constraints (cf. e.g., [2, 11, 33, 36, 37, 38, 39, 40]). However, so far an a posteriori error analysis of adaptive finite element approximations has not been provided for mixed control-state constrained control problems.

In this paper, we will be concerned with the development, analysis and implementation of a residual type a posteriori error estimator for mixed control-state constrained distributed optimal control problems for linear second order elliptic boundary value problems. The paper is organized as follows: In section 2, as a model problem we consider a distributed optimal control problem for a two-dimensional, second order elliptic PDE with a quadratic objective functional and mixed unilateral constraints on the state and on the control. The optimality conditions are stated in terms of the state, the adjoint state, the control, and a Lagrangian multiplier for the mixed constraints. It is, however, not possible to use the residual-type a posteriori error analysis for pure control constrained problems, since the reliability and efficiency estimates involve constants that blow up when the regularization parameter goes to zero. Instead, one has to adopt the error analysis as developed for the pure state constrained case [26]. In this spirit, we further consider a regularized multiplier and a regularized adjoint state which will play an essential role in the error analysis. In section 3, we describe the finite element discretization of the control problem with respect to a family of shape regular simplicial triangulations of the computational domain using continuous, piecewise linear finite elements for the state, the control, and for the adjoint and the regularized adjoint state. In section 4, we present the residual-type a posteriori error estimator for the global discretization errors in the state, the regularized adjoint state, and the control. Data oscillations and consistency errors are considered as well, since they enter the subsequent error analysis. In section 5, we prove reliability of the error estimator, i.e., we show that it gives rise to an upper bound for the global discretization errors up to data oscillations and consistency errors. Section 6 is devoted to the efficiency of the 
estimator by showing that, modulo data oscillations, the error estimator also provides a lower bound for the discretization errors. Finally, section 7 contains a documentation of numerical results for two representative test examples in terms of the convergence history of the adaptive finite element process.

\section{ThE MIXED CONTROL-STATE CONSTRAINED DISTRIBUTED CONTROL PROBLEM}

Let $\Omega$ be a bounded domain in $\mathbb{R}^{2}$ with boundary $\Gamma:=\Gamma_{D} \cup \Gamma_{N}, \Gamma_{D} \cap$ $\Gamma_{N}=\emptyset$. We use standard notation from Lebesgue and Sobolev space theory. In particular, we refer to $L^{2}(\Omega)$ as the Hilbert space of square integrable functions with inner product $(\cdot, \cdot)_{0, \Omega}$ and associated norm $\|\cdot\|_{0, \Omega}$. We denote by $L_{+}^{2}(\Omega)$ the non-negative cone of $L^{2}(\Omega)$, i.e., $L_{+}^{2}(\Omega):=\left\{v \in L^{2}(\Omega) \mid v(x) \geq 0\right.$ f.a.a. $\left.x \in \Omega\right\}$. Moreover, $H^{k}(\Omega), k \in$ $\mathbb{N}$, stands for the Sobolev space of square integrable functions whose weak derivatives up to order $k$ are square integrable as well, equipped with the norm $\|\cdot\|_{k, \Omega}$. $H_{0}^{k}(\Omega)$ denotes its subspace $H_{0}^{k}(\Omega):=\{v \in$ $\left.H^{k}(\Omega)\left|D^{\alpha} v\right|_{\Gamma}=0,|\alpha| \leq k-1\right\}$ and $H^{-k}(\Omega)$ is the dual of $H_{0}^{k}(\Omega)$.

For given $c \in \mathbb{R}_{+}$, we refer to $A: V \rightarrow H^{-1}(\Omega), V:=\{v \in$ $\left.H^{1}(\Omega)|v|_{\Gamma_{D}}=0\right\}$, as the linear second order elliptic differential operator

$$
A y:=-\Delta y+c y \quad, \quad y \in V
$$

and to $a(\cdot, \cdot): V \times V \rightarrow \mathbb{R}$ with $a(y, v):=\int_{\Omega}(\nabla y \cdot \nabla v+c y v) d x$ as the associated bilinear form. We assume $c>0$ or meas $\left(\Gamma_{D}\right) \neq 0$. In particular, this assures that $A$ is bounded and $V$-elliptic, i.e., there exist constants $C>0$ and $\gamma>0$ such that

$$
|a(y, v)| \leq C\|y\|_{1, \Omega}\|v\|_{1, \Omega} \quad, \quad a(y, y) \geq \gamma\|y\|_{1, \Omega}^{2} .
$$

Now, given a desired state $y^{d} \in L^{2}(\Omega)$, a shift control $u^{d} \in L^{2}(\Omega)$, regularization parameters $\alpha>0, \varepsilon>0$, and a function $\psi \in L^{\infty}(\Omega)$, we consider the objective functional

$$
J(y, u):=\frac{1}{2}\left\|y-y^{d}\right\|_{0, \Omega}^{2}+\frac{\alpha}{2}\left\|u-u^{d}\right\|_{0, \Omega}^{2}
$$

and the mixed control-state constrained distributed optimal control problem: Find $(y, u) \in V \times L^{2}(\Omega)$ such that

$$
\inf _{y, u} J(y, u),
$$


subject to the constraints

$$
\begin{aligned}
& A y=u \quad \text { in } \Omega, y=0 \quad \text { on } \Gamma_{D}, \boldsymbol{\nu} \cdot \nabla y=0 \quad \text { on } \Gamma_{N}, \\
& \varepsilon u+y \in K:=\left\{v \in L^{2}(\Omega) \mid v(x) \leq \psi(x) \text { f.a.a. } x \in \Omega\right\} .
\end{aligned}
$$

The usual way to look at $(2.3 \mathrm{a})-(2.3 \mathrm{c})$ is as a regularized state constrained problem, since the multiplier associated with the inequality constraint $(2.3 \mathrm{c})$, usually called the adjoint control for control constrained problems, lives in the non-negative cone of $L^{2}(\Omega)$ and not in a measure space as in the case of pure state constraints. Obviously, the latter case is much more difficult to handle.

We define $G: L^{2}(\Omega) \rightarrow V$ as the control-to-state map which assigns to $u \in L^{2}(\Omega)$ the unique solution $y=y(u)$ of $(2.3 \mathrm{~b})$. We note that the control-to-state map $G$ is a bounded linear operator. Substituting the state $y=y(u)$ by $y(u)=G u$ leads to the reduced objective functional

$$
J_{\text {red }}(u):=\frac{1}{2}\left\|G u-y^{d}\right\|_{0, \Omega}^{2}+\frac{\alpha}{2}\left\|u-u^{d}\right\|_{0, \Omega}^{2},
$$

and the mixed control-state constrained problem (2.3a)-(2.3c) can be restated as

$$
\inf _{\varepsilon u+G u \in K} J_{\text {red }}(u) .
$$

Standard arguments from convex optimization reveal the existence and uniqueness of a solution. The optimality conditions for the optimal solution $(y, u) \in V \times L^{2}(\Omega)$ are as follows.

Theorem 2.1. The optimal solution $(y, u) \in V \times L^{2}(\Omega)$ of $(2.3)$ is characterized by the existence of an adjoint state $p \in V$, and a multiplier $\sigma \in L_{+}^{2}(\Omega)$ such that

$$
\begin{aligned}
(\nabla y, \nabla v)_{0, \Omega}+(c y, v)_{0, \Omega} & =(u, v)_{0, \Omega}, v \in V, \\
(\nabla p, \nabla w)_{0, \Omega}+(c p, w)_{0, \Omega} & =\left(y-y^{d}, w\right)_{0, \Omega}+(\sigma, w)_{0, \Omega}, w \in V, \\
p+\alpha\left(u-u^{d}\right)+\varepsilon \sigma & =0, \\
(\sigma, \varepsilon u+y-\psi)_{0, \Omega} & =0 .
\end{aligned}
$$

The residual-type a posteriori error analysis known from the pure control constrained case [21] is not applicable to (2.6a)-(2.6d) uniformly in the regularization parameter $\varepsilon$, since the constants in the reliability and efficiency estimates for the associated error estimator depend on $\varepsilon$ in the sense that they blow up as $\varepsilon \rightarrow 0$. Instead, the a posteriori error analysis has to be adopted to that what is known from the pure state 
constrained case. Following [26], we define a regularized multiplier $\bar{\sigma} \in V$ as the solution of

$$
(\nabla \bar{\sigma}, \nabla v)_{0, \Omega}+(c \bar{\sigma}, v)_{0, \Omega}=(\sigma, v)_{0, \Omega} \quad, \quad v \in V,
$$

and further introduce a regularized adjoint state $\bar{p} \in V$ according to

$$
(\nabla \bar{p}, \nabla v)_{0, \Omega}+(c \bar{p}, v)_{0, \Omega}=\left(y-y^{d}, v\right)_{0, \Omega}, v \in V .
$$

Obviously, $p, \bar{p}$, and $\bar{\sigma}$ are related by

$$
p:=\bar{p}+\bar{\sigma}
$$

\section{Finite element approximation}

We consider a family $\left\{\mathcal{T}_{\ell}(\Omega)\right\}$ of shape-regular simplicial triangulations of $\Omega$ which align with $\Gamma_{D}, \Gamma_{N}$ on $\Gamma$. We denote by $\mathcal{N}_{\ell}(D)$ and $\mathcal{E}_{\ell}(D), D \subseteq \bar{\Omega}$, the sets of vertices and edges of $\mathcal{T}_{\ell}(\Omega)$ in $D \subseteq \bar{\Omega}$, and we refer to $h_{T}$ and $|T|$ as the diameter and the area of an element $T \in \mathcal{T}_{\ell}(\Omega)$, whereas $h_{E}$ stands for the length of an edge $E \in \mathcal{E}_{\ell}(D)$. For $E \in \mathcal{E}_{\ell}(\Omega)$ such that $E=T_{+} \cap T_{-}, T_{ \pm} \in \mathcal{T}_{\ell}(\Omega)$, we define $\omega_{E}:=T_{+} \cup T_{-}$. For $T \in \mathcal{T}_{\ell}(\Omega)$ and $E \in \mathcal{E}_{\ell}(\Omega)$ we further denote by $\lambda_{i}(T), 1 \leq i \leq 3$, and $\lambda_{i}(E), 1 \leq i \leq 2$, the barycentric coordinates associated with the vertices of $T$ and $E$, respectively. We will also use the following notation: If $A$ and $B$ are two quantities, then $A \lesssim B$ means that there exists a positive constant $C$ such that $A \leq C B$, where $C$ only depends on the shape regularity of the triangulations, but not on their granularities.

The mixed control-state constrained optimal control problem (2.3a)(2.3c) is discretized by continuous piecewise linear finite elements with respect to the triangulation $\mathcal{T}_{\ell}(\Omega)$. In particular, we refer to $S_{\ell}:=$ $\left\{v_{\ell} \in C_{0}(\Omega)\left|v_{\ell}\right|_{T} \in P_{1}(T), T \in \mathcal{T}_{\ell}(\Omega)\right\}$ as the finite element space spanned by the canonical nodal basis functions $\varphi_{\ell}^{p}, p \in \mathcal{N}_{\ell}(\Omega)$, associated with the nodal points in $\bar{\Omega}$ and to $V_{\ell}$ as its subspace $V_{\ell}:=\left\{v_{\ell} \in\right.$ $\left.S_{\ell}\left|v_{\ell}\right|_{\Gamma_{D}}=0\right\}$.

Given some approximation $u_{\ell}^{d} \in S_{\ell}$ of $u^{d}$ and $\psi_{\ell} \in S_{\ell}$ of $\psi$, we refer to $J_{\ell}: V_{\ell} \times S_{\ell} \rightarrow \mathbb{R}$ as the discrete objective functional

$$
J_{\ell}\left(y_{\ell}, u_{\ell}\right):=\frac{1}{2}\left\|y_{\ell}-y^{d}\right\|_{0, \Omega}^{2}+\frac{\alpha}{2}\left\|u_{\ell}-u_{\ell}^{d}\right\|_{0, \Omega}^{2} .
$$

The finite element approximation of the mixed control-state constrained optimal control problem $(2.3 \mathrm{a})-(2.3 \mathrm{c})$ reads as follows:

Find $\left(y_{\ell}, u_{\ell}\right) \in V_{\ell} \times S_{\ell}$ such that

$$
\inf _{y_{\ell}, u_{\ell}} J_{\ell}\left(y_{\ell}, u_{\ell}\right)
$$


subject to the constraints

$$
\begin{gathered}
\left(\nabla y_{\ell}, \nabla v_{\ell}\right)_{0, \Omega}+\left(c y_{\ell}, v_{\ell}\right)_{0, \Omega}=\left(u_{\ell}, v_{\ell}\right)_{0, \Omega} \quad, \quad v_{\ell} \in V_{\ell} \\
\varepsilon u_{\ell}+y_{\ell} \in K_{\ell}:=\left\{v_{\ell} \in S_{\ell} \mid v_{\ell} \leq \psi_{\ell}\right\}
\end{gathered}
$$

As in the continuous setting, the discrete state constrained optimal control problem (3.2a)-(3.2c) admits a unique solution $\left(y_{\ell}, u_{\ell}\right) \in V_{\ell} \times S_{\ell}$. We obtain the discrete optimality conditions:

Theorem 3.1. Let $\left(y_{\ell}, u_{\ell}\right) \in V_{\ell} \times S_{\ell}$ be the unique solution of (3.2a)(3.2c). Then, there exist a discrete adjoint state $p_{\ell} \in V_{\ell}$ as well as a discrete multiplier $\sigma_{\ell} \in V_{\ell} \cap L_{+}^{2}(\Omega)$ such that

$$
\begin{aligned}
\left(\nabla y_{\ell}, \nabla v_{\ell}\right)_{0, \Omega}+\left(c y_{\ell}, v_{\ell}\right)_{0, \Omega}= & \left(u_{\ell}, v_{\ell}\right)_{0, \Omega}, v_{\ell} \in V_{\ell} \\
\left(\nabla p_{\ell}, \nabla v_{\ell}\right)_{0, \Omega}+\left(c p_{\ell}, v_{\ell}\right)_{0, \Omega}= & \left(y_{\ell}-y^{d}, v_{\ell}\right)_{0, \Omega}+ \\
& +\left(\sigma_{\ell}, v_{\ell}\right)_{0, \Omega}, v_{\ell} \in V_{\ell} \\
p_{\ell}+\alpha\left(u_{\ell}-u_{\ell}^{d}\right)+\varepsilon \sigma_{\ell}= & 0 \\
\left(\sigma_{\ell}, \varepsilon u_{\ell}+y_{\ell}-\psi_{\ell}\right)_{0, \Omega}= & 0 .
\end{aligned}
$$

As in section 2 before, we introduce a regularized discrete multiplier $\bar{\sigma}_{\ell} \in V_{\ell}$ as the solution of

$$
\left(\nabla \bar{\sigma}_{\ell}, \nabla v_{\ell}\right)_{0, \Omega}+\left(c \bar{\sigma}_{\ell}, v_{\ell}\right)_{0, \Omega}=\left(\sigma_{\ell}, v_{\ell}\right)_{0, \Omega}, v_{\ell} \in V_{\ell}
$$

and define $\bar{p}_{\ell} \in V_{\ell}$ as the solution of the discrete analogue of (2.8), i.e.,

$$
\left(\nabla \bar{p}_{\ell}, \nabla v_{\ell}\right)_{0, \Omega}+\left(c \bar{p}_{\ell}, v_{\ell}\right)_{0, \Omega}=\left(y_{\ell}-y^{d}, v_{\ell}\right)_{0, \Omega}, v_{\ell} \in V_{\ell}
$$

As in the continuous setting, we obtain the fundamental relationship

$$
p_{\ell}=\bar{p}_{\ell}+\bar{\sigma}_{\ell}
$$

We further define $\mathcal{A}\left(y_{\ell}\right)$ and $\mathcal{I}\left(y_{\ell}\right)$ as the discrete active and inactive sets according to

$$
\begin{aligned}
\mathcal{A}\left(y_{\ell}\right) & :=\bigcup\left\{T \in \mathcal{T}_{\ell}(\Omega) \mid y_{\ell}(p)=\psi_{\ell}(p), p \in \mathcal{N}_{\ell}(T)\right\} \\
\mathcal{I}\left(y_{\ell}\right) & :=\mathcal{T}_{\ell}(\Omega) \backslash \mathcal{A}\left(y_{\ell}\right) .
\end{aligned}
$$

\section{THE RESIDUAL TYPE ERROR ESTIMATOR}

The residual-type a posteriori error estimator involves estimators of the state $y$ and of the regularized adjoint state $\bar{p}$ according to

$$
\eta_{\ell}:=\eta_{\ell}(y)+\eta_{\ell}(\bar{p})
$$


where $\eta_{\ell}(y)$ and $\eta_{\ell}(\bar{p})$ consist of element and edge residuals

$$
\begin{aligned}
& \eta_{\ell}(y):=\left(\sum_{T \in \mathcal{T}_{\ell}(\Omega)} \eta_{T}^{2}(y)+\sum_{E \in \mathcal{E}_{\ell}(\Omega)} \eta_{E}^{2}(y)\right)^{1 / 2} \\
& \eta_{\ell}(\bar{p}):=\left(\sum_{T \in \mathcal{T}_{\ell}(\Omega)} \eta_{T}^{2}(\bar{p})+\sum_{E \in \mathcal{E}_{\ell}(\Omega)} \eta_{E}^{2}(\bar{p})\right)^{1 / 2} .
\end{aligned}
$$

The element residuals $\eta_{T}(y)$ and $\eta_{T}(\bar{p}), T \in \mathcal{T}_{\ell}(\Omega)$, are weighted elementwise $L^{2}$-residuals with respect to the strong form of the state equation (2.3b) and the modified adjoint state equation (2.8), respectively:

$$
\begin{aligned}
& \eta_{T}(y):=h_{T}\left\|c y_{\ell}-u_{\ell}\right\|_{0, T}, T \in \mathcal{T}_{\ell}(\Omega), \\
& \eta_{T}(\bar{p}):=h_{T}\left\|c \bar{p}_{\ell}-\left(y_{\ell}-y^{d}\right)\right\|_{0, T}, T \in \mathcal{T}_{\ell}(\Omega) .
\end{aligned}
$$

The edge residuals $\eta_{E}(y)$ and $\eta_{E}(\bar{p}), E \in \mathcal{E}_{\ell}(\Omega)$, are weighted $L^{2}$-norms of the jumps $\nu_{E} \cdot\left[\nabla y_{\ell}\right]$ and $\nu_{E} \cdot\left[\nabla \bar{p}_{\ell}\right]$ of the normal derivatives across the interior edges

$$
\begin{aligned}
& \eta_{E}(y):=h_{E}^{1 / 2}\left\|\nu_{E} \cdot\left[\nabla y_{\ell}\right]\right\|_{0, E}, E \in \mathcal{E}_{\ell}(\Omega), \\
& \eta_{E}(\bar{p}):=h_{E}^{1 / 2}\left\|\nu_{E} \cdot\left[\nabla \bar{p}_{\ell}\right]\right\|_{0, E}, E \in \mathcal{E}_{\ell}(\Omega) .
\end{aligned}
$$

Denoting by $y_{\ell}^{d} \in S_{\ell}$ some approximation of the desired state $y^{d}$, we further have to take into account data oscillations with respect to the data $u^{d}, y^{d}, \psi$ of the problem

$$
o s c_{\ell}:=\left(\operatorname{osc}_{\ell}^{2}\left(u^{d}\right)+\operatorname{osc}_{\ell}^{2}\left(y^{d}\right)+\operatorname{osc}_{\ell}^{2}(\psi)\right)^{1 / 2}
$$

where $\operatorname{osc}_{\ell}\left(u^{d}\right), \operatorname{osc}_{\ell}\left(y^{d}\right)$, and $\operatorname{ssc}_{\ell}(\psi)$ are given by

$$
\begin{aligned}
& \operatorname{osc}_{\ell}\left(u^{d}\right):=\left(\sum_{T \in \mathcal{T}_{\ell}(\Omega)} \operatorname{osc}_{T}^{2}\left(u^{d}\right)\right)^{1 / 2}, \\
& \operatorname{osc}_{T}\left(u^{d}\right):=\left\|u^{d}-u_{\ell}^{d}\right\|_{0, T}, T \in \mathcal{T}_{\ell}(\Omega), \\
& \operatorname{osc}_{\ell}\left(y^{d}\right):=\left(\sum_{T \in \mathcal{T}_{\ell}(\Omega)} \operatorname{osc}_{T}^{2}\left(y^{d}\right)\right)^{1 / 2}, \\
& \operatorname{osc}_{T}\left(y^{d}\right):=h_{T}\left\|y^{d}-y_{\ell}^{d}\right\|_{0, T}, T \in \mathcal{T}_{\ell}(\Omega), \\
& \operatorname{osc}_{\ell}(\psi):=\left(\sum_{T \in \mathcal{T}_{\ell}(\Omega)} \operatorname{osc}_{T}^{2}(\psi)\right)^{1 / 2}, \\
& \operatorname{osc}_{T}(\psi):=\left\|\psi-\psi_{\ell}\right\|_{0, T}, T \in \mathcal{T}_{\ell}(\Omega) .
\end{aligned}
$$

We will show that, up to data oscillations and consistency errors, the residual-type a posteriori error estimator (4.1) provides an upper and 
a lower bound for the discretization errors in the state, the regularized adjoint state, and the control which are given by

$$
e_{y}:=y-y_{\ell} \quad, \quad e_{\bar{p}}:=\bar{p}-\bar{p}_{\ell} \quad, \quad e_{u}:=u-u_{\ell} .
$$

In much the same way as in case of adaptive finite element discretizations of pure state constrained elliptic boundary value problems (cf. $[26])$, the a posteriori error analysis requires an auxiliary state $y\left(u_{\ell}\right) \in$ $V$ and an auxiliary adjoint state $\bar{p}\left(y_{\ell}\right) \in V$. These are defined as the solutions of the following variational equations

$$
\begin{aligned}
& \left(\nabla y\left(u_{\ell}\right), \nabla v\right)_{0, \Omega}+\left(c y\left(u_{\ell}\right), v\right)_{0, \Omega}=\left(u_{\ell}, v\right)_{0, \Omega}, v \in V, \\
& \left(\nabla \bar{p}\left(y_{\ell}\right), \nabla v\right)_{0, \Omega}+\left(c \bar{p}\left(y_{\ell}\right), v\right)_{0, \Omega}=\left(y_{\ell}-y^{d}, v\right)_{0, \Omega}, v \in V .
\end{aligned}
$$

We further introduce an auxiliary discrete state $y_{\ell}(u) \in V_{\ell}$ as the solution of the finite dimensional variational problem

$$
\left(\nabla y_{\ell}(u), \nabla v_{\ell}\right)_{0, \Omega}+\left(c y_{\ell}(u), v_{\ell}\right)_{0, \Omega}=\left(u, v_{\ell}\right)_{0, \Omega}, v_{\ell} \in V_{\ell}
$$

The auxiliary states $y\left(u_{\ell}\right) \in V$ and $y_{\ell}(u) \in V_{\ell}$ do not necessarily satisfy the state constraints, i.e., it may happen that $\varepsilon u+y\left(u_{\ell}\right) \notin K$ or $\varepsilon u_{\ell}+$ $y_{\ell}(u) \notin K_{\ell}$. Therefore, we introduce the consistency errors

$$
\begin{aligned}
e_{c}^{(1)}\left(\psi, \psi_{\ell}\right):= & \max \left(\left(\sigma-\sigma_{\ell}, \psi-\psi_{\ell}\right)_{0, \Omega}, 0\right) \\
e_{c}^{(2)}\left(u, u_{\ell}\right):= & \max \left(\left(\sigma_{\ell}, \varepsilon u_{\ell}+y_{\ell}(u)-\psi_{\ell}\right)_{0, \Omega}+\right. \\
& \left.+\left(\sigma, \varepsilon u+y\left(u_{\ell}\right)-\psi\right)_{0, \Omega}, 0\right) .
\end{aligned}
$$

Obviously, $e_{c}^{(1)}\left(\psi, \psi_{\ell}\right)=0$ for $\psi=\psi_{\ell}$. Moreover, we note that for $u=$ $u_{\ell}$ we have $e_{c}^{(2)}\left(u, u_{\ell}\right)=0$, since in this case $y\left(u_{\ell}\right)=y$ and $y_{\ell}(u)=y_{\ell}$, and hence, $e_{c}^{(2)}\left(u, u_{\ell}\right)=0$ due to $(2.6 \mathrm{~d})$ and $(3.3 \mathrm{~d})$. We thus define

$$
\begin{gathered}
\tilde{e}_{c}^{(1)}\left(\psi, \psi_{\ell}\right):=\left\{\begin{array}{r}
e_{c}^{(1)}\left(\psi, \psi_{\ell}\right) /\left\|\psi-\psi_{\ell}\right\|_{0, \Omega}, \psi \neq \psi_{\ell} \\
0, \psi=\psi_{\ell}
\end{array}\right. \\
\tilde{e}_{c}^{(2)}\left(u, u_{\ell}\right):=\left\{\begin{array}{r}
e_{c}^{(2)}\left(u, u_{\ell}\right) /\left\|u-u_{\ell}\right\|_{0, \Omega}, u \neq u_{\ell} \\
0, u=u_{\ell}
\end{array}\right.
\end{gathered}
$$

The refinement of a triangulation $\mathcal{T}_{\ell}(\Omega)$ is based on bulk criteria that have been previously used in the convergence analysis of adaptive finite element for nodal finite element methods $[13,34]$. For the mixed control-state constrained optimal control problem under consideration, the bulk criteria are as follows: Given universal constants $\Theta_{i} \in(0,1), 1 \leq$ $i \leq 5$, we create a set of edges $\mathcal{M}_{E} \subset \mathcal{E}_{h}(\Omega)$ and sets of elements $\mathcal{M}_{\eta, T}, \mathcal{M}_{u^{d}, T}, \mathcal{M}_{y^{d}, T}, \mathcal{M}_{\psi, T} \subset \mathcal{T}_{\ell}(\Omega)$ such that

$$
\Theta_{1} \sum_{E \in \mathcal{E}_{\ell}(\Omega)}\left(\eta_{E}^{2}(y)+\eta_{E}^{2}(\bar{p})\right) \leq \sum_{E \in \mathcal{M}_{E}}\left(\eta_{E}^{2}(y)+\eta_{E}^{2}(\bar{p})\right),
$$




$$
\begin{aligned}
\Theta_{2} \sum_{T \in \mathcal{T}_{\ell}(\Omega)}\left(\eta_{T}^{2}(y)+\eta_{T}^{2}(\bar{p})\right) & \leq \sum_{T \in \mathcal{M}_{\eta, T}}\left(\eta_{T}^{2}(y)+\eta_{T}^{2}(\bar{p})\right), \\
\Theta_{3} \sum_{T \in \mathcal{T}_{\ell}(\Omega)} o s c_{T}^{2}\left(u^{d}\right) & \leq \sum_{T \in \mathcal{M}_{u^{d}, T}} \operatorname{osc}_{T}^{2}\left(u^{d}\right) \\
\Theta_{4} \sum_{T \in \mathcal{T}_{\ell}(\Omega)}{ }_{\operatorname{ssc}}^{2}\left(y^{d}\right) & \leq \sum_{T \in \mathcal{M}_{y^{d}, T}} \operatorname{osc}_{T}^{2}\left(y^{d}\right) \\
\Theta_{5} \sum_{T \in \mathcal{T}_{\ell}(\Omega)} \operatorname{osc}_{T}^{2}(\psi) & \leq \sum_{T \in \mathcal{M}_{\psi, T}} \operatorname{osc}_{T}^{2}(\psi) .
\end{aligned}
$$

The bulk criteria are realized by a greedy algorithm (cf., e.g., [21]). We set

$$
\mathcal{M}_{T}:=\mathcal{M}_{\eta, T} \cup \mathcal{M}_{u^{d}, T} \cup \mathcal{M}_{y^{d}, T} \cup \mathcal{M}_{\psi, T}
$$

and refine an element $T \in \mathcal{T}_{\ell}(\Omega)$ by bisection (i.e., by joining the midpoint of the longest edge with the opposite vertex), if $T \in \mathcal{M}^{T}$ and an edge $E \in \mathcal{E}_{\ell}(T)$ by bisection (joining its midpoint with the opposite vertices of the adjacent elements), if $E \in \mathcal{M}^{E}$.

Denoting by $\mathcal{N}_{T}:=\left\{T^{\prime} \in \mathcal{T}_{\ell}(\Omega) \mid T^{\prime} \cap T \neq \emptyset\right\}$ the set of all neighboring triangles of $T \in \mathcal{T}_{\ell}(\Omega)$, we define the set

$$
\mathcal{F}_{\ell}\left(y_{\ell}\right):=\partial \mathcal{A}\left(y_{\ell}\right) \cup \partial \mathcal{I}\left(y_{\ell}\right)
$$

where

$$
\begin{aligned}
\partial \mathcal{A}\left(y_{\ell}\right) & :=\bigcup\left\{T \subset \mathcal{A}\left(y_{\ell}\right) \mid \mathcal{N}_{T} \cap \mathcal{I}\left(y_{\ell}\right) \neq \emptyset\right\} \\
\partial \mathcal{I}\left(y_{\ell}\right) & :=\bigcup\left\{T \subset \mathcal{I}\left(y_{\ell}\right) \mid \mathcal{N}_{T} \cap \mathcal{A}\left(y_{\ell}\right) \neq \emptyset\right\} .
\end{aligned}
$$

The set $\mathcal{F}_{\ell}\left(y_{\ell}\right)$ represents a neighborhood of the discrete free boundary between the discrete active and inactive sets $\mathcal{A}\left(y_{\ell}\right)$ and $\mathcal{I}\left(y_{\ell}\right)$. In order to guarantee a sufficient resolution of the continuous free boundary between $\mathcal{A}(y)$ and $\mathcal{I}(y)$, at each refinement step, the elements $T \in$ $\mathcal{F}_{\ell}\left(u_{\ell}\right)$ are refined by bisection.

\section{Reliability OF THE ERROR ESTIMATOR}

We prove reliability of the residual-type error estimator (4.1) in the sense that it provides an upper bound for the discretization errors $e_{y}, e_{u}$, and $e_{\bar{p}}$ up to the data oscillations $\operatorname{osc}_{\ell}\left(u^{d}\right)$ and $\operatorname{osc}_{\ell}(\psi)$ and the consistency errors $\tilde{e}_{c}^{(2)}\left(u, u_{\ell}\right)$ and $\tilde{e}_{c}^{(1)}\left(\psi, \psi_{\ell}\right)$.

Theorem 5.1. Let $(y, u, p, \sigma)$ and $\left(y_{\ell}, u_{\ell}, p_{\ell}, \sigma_{\ell}\right)$ be the solutions of (2.6a)-(2.6d) and (3.3a)-(3.3d) and let $\eta_{\ell}, \operatorname{osc}_{\ell}\left(u^{d}\right)$ and $\tilde{e}_{c}^{(2)}\left(u, u_{\ell}\right)$ as well as $\tilde{e}_{c}^{(1)}\left(\psi, \psi_{\ell}\right)$ be the error estimator, the data oscillation in the shift 
control, and the consistency errors according to (4.1),(4.6) and (4.11), respectively. Further, let $\bar{p}$ and $\bar{p}_{\ell}$ be the regularized adjoint states as given by (2.8),(3.5). Then, there holds

$$
\begin{aligned}
& \left\|e_{y}\right\|_{1, \Omega}+\left\|e_{u}\right\|_{0, \Omega}+\left\|e_{\bar{p}}\right\|_{1, \Omega} \lesssim \\
& \lesssim \eta+\operatorname{osc}_{\ell}\left(u^{d}\right)+\operatorname{osc}_{\ell}(\psi)+\tilde{e}_{c}^{(1)}\left(\psi, \psi_{\ell}\right)+\tilde{e}_{c}^{(2)}\left(u, u_{\ell}\right) .
\end{aligned}
$$

The proof of Theorem 5.1 will be given by the following three Lemmas $5.2,5.3$ and 5.4 .

Lemma 5.2. In addition to the assumptions of Theorem 5.1 let $y\left(u_{\ell}\right)$ and $\bar{p}\left(y_{\ell}\right)$ be the auxiliary state and auxiliary adjoint state according to $(4.8 \mathrm{a}),(4.8 \mathrm{~b})$. Then, there holds

$$
\left\|e_{y}\right\|_{1, \Omega}+\left\|e_{\bar{p}}\right\|_{1, \Omega} \lesssim\left\|y\left(u_{\ell}\right)-y_{\ell}\right\|_{1, \Omega}+\left\|\bar{p}\left(y_{\ell}\right)-\bar{p}_{\ell}\right\|_{1, \Omega}+\left\|e_{u}\right\|_{0, \Omega}
$$

Proof. Obviously, $e_{y}$ and $e_{\bar{p}}$ can be estimated from above by

$$
\begin{aligned}
& \left\|e_{y}\right\|_{1, \Omega} \leq\left\|y-y\left(u_{\ell}\right)\right\|_{1, \Omega}+\left\|y\left(u_{\ell}\right)-y_{\ell}\right\|_{1, \Omega}, \\
& \left\|e_{\bar{p}}\right\|_{1, \Omega} \leq\left\|\bar{p}-\bar{p}\left(y_{\ell}\right)\right\|_{1, \Omega}+\left\|\bar{p}\left(y_{\ell}\right)-\bar{p}_{\ell}\right\|_{1, \Omega} .
\end{aligned}
$$

Setting $v=y-y\left(u_{\ell}\right)$ in (2.6a),(4.8a), and $M:=1 / \gamma$ with $\gamma$ from (2.1), for the first term on the right-hand side in (5.3a) we readily get

$$
\left\|y-y\left(u_{\ell}\right)\right\|_{1, \Omega}^{2} \leq M\left\|e_{u}\right\|_{0, \Omega}\left\|y-y\left(u_{\ell}\right)\right\|_{0, \Omega} \leq M\left\|e_{u}\right\|_{0, \Omega}\left\|y-y\left(u_{\ell}\right)\right\|_{1, \Omega},
$$

and hence,

$$
\left\|y-y\left(u_{\ell}\right)\right\|_{1, \Omega} \leq M\left\|e_{u}\right\|_{0, \Omega} .
$$

Likewise, choosing $v=\bar{p}-\bar{p}\left(y_{\ell}\right)$ in (2.8) and (4.8b), for the first term on the right-hand side in $(5.3 \mathrm{~b})$ it follows that

$$
\left\|\bar{p}-\bar{p}\left(y_{\ell}\right)\right\|_{1, \Omega}^{2} \leq M\left\|e_{y}\right\|_{0, \Omega}\left\|\bar{p}-\bar{p}\left(y_{\ell}\right)\right\|_{0, \Omega} \leq M\left\|e_{y}\right\|_{1, \Omega}\left\|\bar{p}-\bar{p}\left(y_{\ell}\right)\right\|_{1, \Omega} .
$$

Consequently, in view of (5.3a) and (5.4) we obtain

$$
\left\|\bar{p}-\bar{p}\left(y_{\ell}\right)\right\|_{1, \Omega} \leq M\left\|e_{y}\right\|_{1, \Omega} \leq M^{2}\left\|e_{u}\right\|_{0, \Omega}+M\left\|y\left(u_{\ell}\right)-y_{\ell}\right\|_{1, \Omega} .
$$

Using $(5.4),(5.5)$ in $(5.3 \mathrm{a}),(5.3 \mathrm{~b})$ gives $(5.2)$.

Lemma 5.3. Under the same assumptions as in Lemma 5.2 there holds

$$
\begin{aligned}
& \left\|e_{u}\right\|_{0, \Omega}^{2} \leq \frac{3}{\alpha^{2}}\left(M^{2}\left\|y\left(u_{\ell}\right)-y_{\ell}\right\|_{1, \Omega}^{2}+\left\|\bar{p}\left(y_{\ell}\right)-\bar{p}_{\ell}\right\|_{1, \Omega}^{2}+\right. \\
& \left.+\left(\tilde{e}_{c}^{(1)}\left(\psi, \psi_{\ell}\right)\right)^{2}+\left(\tilde{e}_{c}^{(2)}\left(u, u_{\ell}\right)\right)^{2}+\operatorname{osc}_{\ell}^{2}\left(u^{d}\right)\right)+\frac{1}{3} \operatorname{osc}_{\ell}^{2}(\psi) .
\end{aligned}
$$


Proof. Using (2.6c),(2.9) and (3.3c),(3.6), we find

$$
\begin{aligned}
\alpha\left\|e_{u}\right\|_{0, \Omega}^{2}= & \left(e_{u}, \bar{p}_{\ell}-\bar{p}\right)_{0, \Omega}+\varepsilon\left(e_{u}, \sigma_{\ell}-\sigma\right)_{0, \Omega}+ \\
& +\left(e_{u}, \bar{\sigma}_{\ell}-\bar{\sigma}\right)_{0, \Omega}+\alpha\left(e_{u}, u^{d}-u_{\ell}^{d}\right)_{0, \Omega} .
\end{aligned}
$$

The first term on the right-hand side in (5.7) can be split according to

$$
\left(e_{u}, \bar{p}_{\ell}-\bar{p}\right)_{0, \Omega}=\left(e_{u}, \bar{p}_{\ell}-\bar{p}\left(y_{\ell}\right)\right)_{0, \Omega}+\left(e_{u}, \bar{p}\left(y_{\ell}\right)-\bar{p}\right)_{0, \Omega}
$$

An application of Young's inequality yields

$$
\left(e_{u}, \bar{p}_{\ell}-\bar{p}\left(y_{\ell}\right)\right)_{0, \Omega} \leq \frac{\alpha}{12}\left\|e_{u}\right\|_{0, \Omega}^{2}+\frac{3}{\alpha}\left\|\bar{p}_{\ell}-\bar{p}\left(y_{\ell}\right)\right\|_{0, \Omega}^{2}
$$

On the other hand, choosing $v=\bar{p}-\bar{p}\left(y_{\ell}\right)$ in (2.6a),(4.8a) and $v=$ $y-y\left(u_{\ell}\right)$ in $(2.8),(4.8 \mathrm{~b})$, for the second term on the right-hand side in (5.8) we get

$$
\begin{aligned}
& \left(e_{u}, \bar{p}\left(y_{\ell}\right)-\bar{p}\right)_{0, \Omega}=-\left(y-y_{\ell}, y-y\left(u_{\ell}\right)\right)_{0, \Omega}= \\
& =-\left\|y-y\left(u_{\ell}\right)\right\|_{0, \Omega}^{2}+\left(y_{\ell}-y\left(u_{\ell}\right), y-y\left(u_{\ell}\right)\right)_{0, \Omega} \leq \\
& \leq\left\|y-y\left(u_{\ell}\right)\right\|_{1, \Omega}\left\|y\left(u_{\ell}\right)-y_{\ell}\right\|_{1, \Omega} \leq \\
& \leq \frac{\alpha}{12}\left\|e_{u}\right\|_{0, \Omega}^{2}+\frac{3 M^{2}}{\alpha}\left\|y\left(u_{\ell}\right)-y_{\ell}\right\|_{1, \Omega}^{2},
\end{aligned}
$$

where we have further made use of (5.4) and of Young's inequality in the last estimate. Using (5.9) and (5.10) in (5.8) results in

$$
\begin{aligned}
& \left(e_{u}, \bar{p}_{\ell}-\bar{p}\left(y_{\ell}\right)\right)_{0, \Omega} \leq \\
& \leq \frac{\alpha}{6}\left\|e_{u}\right\|_{0, \Omega}^{2}+\frac{3}{\alpha}\left(\left\|\bar{p}_{\ell}-\bar{p}\left(y_{\ell}\right)\right\|_{1, \Omega}^{2}+M^{2}\left\|y\left(u_{\ell}\right)-y_{\ell}\right\|_{1, \Omega}^{2}\right) .
\end{aligned}
$$

As far as the third term on the right-hand side in (5.7) is concerned, in view of (2.6a), (3.3a), (4.8a), (4.9), (2.7) and (3.4) we obtain

$$
\begin{aligned}
& \left(e_{u}, \bar{\sigma}_{\ell}-\bar{\sigma}\right)_{0, \Omega}= \\
& =\left(\nabla\left(y_{\ell}(u)-y_{\ell}\right), \nabla \bar{\sigma}_{\ell}\right)_{0, \Omega}+\left(c\left(y_{\ell}(u)-y_{\ell}\right), \bar{\sigma}_{\ell}\right)_{0, \Omega}- \\
& -\left(\nabla\left(y-y\left(u_{\ell}\right)\right), \nabla \bar{\sigma}\right)_{0, \Omega}-\left(c\left(y-y\left(u_{\ell}\right)\right), \bar{\sigma}\right)_{0, \Omega}= \\
& =\left(\sigma_{\ell}, y_{\ell}(u)-y_{\ell}\right)_{0, \Omega}+\left(\sigma, y\left(u_{\ell}\right)-y\right)_{0, \Omega} .
\end{aligned}
$$

Combining (5.12) with the second term on the right-hand side in (5.7) and using the complementarity conditions (2.6d) and (3.3d) as well as 
(4.11a) and (4.11b), we find

$$
\begin{aligned}
& \varepsilon\left(e_{u}, \sigma_{\ell}-\sigma\right)_{0, \Omega}+\left(e_{u}, \bar{\sigma}_{\ell}-\bar{\sigma}\right)_{0, \Omega}=\left(\sigma_{\ell}, \varepsilon u+y_{\ell}(u)-\left(\varepsilon u_{\ell}+y_{\ell}\right)\right)_{0, \Omega} \\
& -\left(\sigma, \varepsilon u+y-\left(\varepsilon u_{\ell}+y\left(u_{\ell}\right)\right)\right)_{0, \Omega}=\left(\sigma_{\ell}, \varepsilon u+y_{\ell}(u)-\psi\right)_{0, \Omega} \\
& +\left(\sigma, \varepsilon u_{\ell}+y\left(u_{\ell}\right)-\psi_{\ell}\right)_{0, \Omega}+\left(\sigma_{\ell}-\sigma, \psi-\psi_{\ell}\right)_{0, \Omega} \\
& +\underbrace{\left(\sigma_{\ell}, \psi_{\ell}-\left(\varepsilon u_{\ell}+y_{\ell}\right)\right)_{0, \Omega}}_{=0}-\underbrace{(\sigma, \varepsilon u+y-\psi)_{0, \Omega}}_{=0} \\
& \leq\left\|u-u_{\ell}\right\|_{0, \Omega} \tilde{e}_{c}^{(2)}\left(u, u_{\ell}\right)+\left\|\psi-\psi_{\ell}\right\|_{0, \Omega} \tilde{e}_{c}^{(1)}\left(\psi, \psi_{\ell}\right),
\end{aligned}
$$

whence by Young's inequality

$$
\begin{aligned}
& \varepsilon\left(e_{u}, \sigma_{\ell}-\sigma\right)_{0, \Omega}+\left(e_{u}, \bar{\sigma}_{\ell}-\bar{\sigma}\right)_{0, \Omega} \leq \\
\leq & \frac{\alpha}{6}\left(\left\|e_{u}\right\|_{0, \Omega}^{2}+\operatorname{osc}_{\ell}^{2}(\psi)\right)+\frac{3}{2 \alpha}\left(\left(\tilde{e}_{c}^{(1)}\left(\psi, \psi_{\ell}\right)\right)^{2}+\left(\tilde{e}_{c}^{(2)}\left(u, u_{\ell}\right)\right)^{2}\right) .
\end{aligned}
$$

Finally, another application of Young's inequality gives us the following upper bound for the fourth term on the right-hand side in (5.7)

$$
\alpha\left(e_{u}, u^{d}-u_{\ell}^{d}\right)_{0, \Omega} \leq \frac{\alpha}{6}\left\|e_{u}\right\|_{0, \Omega}^{2}+\frac{3}{2 \alpha} \operatorname{osc}_{\ell}^{2}\left(u^{d}\right) .
$$

Taking advantage of the estimates $(5.11),(5.13),(5.14)$ in (5.7) allows to conclude.

Lemma 5.4. Under the same assumptions as in Lemma 5.2 there holds

$$
\begin{aligned}
&\left\|y\left(u_{\ell}\right)-y_{\ell}\right\|_{1, \Omega} \lesssim \eta_{y}, \\
&\left\|\bar{p}\left(y_{\ell}\right)-\bar{p}_{\ell}\right\|_{1, \Omega} \lesssim \eta_{\bar{p}} .
\end{aligned}
$$

Proof. Due to Galerkin orthogonality, the assertion follows by standard arguments from the a posteriori error analysis of adaptive finite element methods (see, e.g., [41]).

Proof of Theorem 5.1. Combining the estimates from Lemma 5.2, Lemma 5.3, and Lemma 5.4 results in (5.1).

\section{LOCAL EFFICIENCY OF THE ERROR ESTIMATOR}

Efficiency of the estimator means that up to data oscillations it also provides a lower bound for the discretization errors in the state, the regularized adjoint state, and the control.

Theorem 6.1. Let $(y, u, p, \sigma)$ and $\left(y_{\ell}, u_{\ell}, p_{\ell}, \sigma_{\ell}\right)$ be the solutions of (2.6a)-(2.6d) and (3.3a)-(3.3d) and let $\eta_{\ell}$ and $\operatorname{osc}_{\ell}\left(y^{d}\right)$ be the error 
estimator and the data oscillation as given by (4.1) and (4.6b), respectively. Further, let $\bar{p}$ and $\bar{p}_{\ell}$ be the modified adjoint states as given by (2.8),(3.5). Then, there holds

$$
\eta_{\ell}-\operatorname{osc}_{\ell}\left(y^{d}\right) \lesssim\left\|e_{y}\right\|_{1, \Omega}+\left\|e_{u}\right\|_{0, \Omega}+\left\|e_{\bar{p}}\right\|_{1, \Omega} .
$$

The proof of the efficiency of the estimator is usually done by establishing local efficiency in the sense that the element and edge residuals can be bounded from above by norms of the discretization errors on the elements and associated patches, respectively. Local efficiency will be provided by the subsequent two lemmas.

Lemma 6.2. Let $\eta_{T}(y)$ and $\eta_{T}(\bar{p}), T \in \mathcal{T}_{\ell}(\Omega)$, be the element residuals as given by (4.3). Then, there holds

$$
\begin{aligned}
\eta_{T}^{2}(y) & \lesssim\left\|e_{y}\right\|_{1, T}^{2}+h_{T}^{2}\left\|e_{u}\right\|_{0, T}^{2}, \\
\eta_{T}^{2}(\bar{p}) & \lesssim\left\|e_{\bar{p}}\right\|_{1, T}^{2}+h_{T}^{2}\left\|e_{y}\right\|_{0, T}^{2}+\operatorname{osc}_{T}^{2}\left(y^{d}\right) .
\end{aligned}
$$

Proof. We denote by $\varphi_{T}=\prod_{i=1}^{3} \lambda_{i}(T)$ the element bubble function associated with $T \in \mathcal{T}_{\ell}(\Omega)$ (cf., e.g., [41]). Then, $\xi_{\ell}:=\left(u_{\ell}-c y_{\ell}\right) \varphi_{T}$ is an admissible test function in (2.6a). Observing $\left.\Delta y_{\ell}\right|_{T}=0$, we obtain

$$
\begin{aligned}
& \eta_{T}^{2}(y) \lesssim h_{T}^{2}\left(u_{\ell}-c y_{\ell}, \xi_{\ell}\right)_{0, T}= \\
& =h_{T}^{2}\left(\left(u, \xi_{\ell}\right)_{0, T}+\left(\Delta y_{\ell}-c y_{\ell}, \xi_{\ell}\right)_{0, T}+\left(u_{\ell}-u, \xi_{\ell}\right)_{0, T}\right)= \\
& =h_{T}^{2}\left(\left(\nabla\left(y-y_{\ell}\right), \nabla \xi_{\ell}\right)_{0, T}+\left(c\left(y-y_{\ell}\right), \xi_{\ell}\right)_{0, T}+\left(u_{\ell}-u, \xi_{\ell}\right)_{0, T}\right) .
\end{aligned}
$$

Using standard estimates for $\left\|\nabla \xi_{\ell}\right\|_{0, T}$ and $\left\|\xi_{\ell}\right\|_{0, T}$ (cf., e.g., [41]) readily gives (6.2). The estimate (6.3) can be verified in the same way.

Lemma 6.3. Let $\eta_{T}(y), \eta_{T}(\bar{p}), T \in \mathcal{T}_{\ell}(\Omega)$, and $\eta_{E}(y), \eta_{E}(\bar{p}), E \in \mathcal{E}_{\ell}(\Omega)$, be the element and edge residuals as given by (4.3),(4.4). Further, let $\operatorname{osc}_{T}\left(y^{d}\right), T \in \mathcal{T}_{\ell}(\Omega)$, be the element contribution to the data oscillation in $y^{d}$ according to $(4.6 \mathrm{~b})$. Then, there holds

$$
\begin{aligned}
\eta_{E}^{2}(y) & \lesssim\left\|e_{y}\right\|_{1, \omega_{E}}^{2}+h_{E}^{2}\left\|e_{u}\right\|_{0, \omega_{E}}^{2}+\eta_{\omega_{E}}^{2}(y), \\
\eta_{E}^{2}(\bar{p}) & \lesssim\left\|e_{\bar{p}}\right\|_{1, \omega_{E}}^{2}+h_{E}^{2}\left\|e_{y}\right\|_{0, \omega_{E}}^{2}+\eta_{\omega_{E}}^{2}(\bar{p})+o s c_{\omega_{E}}^{2}\left(y^{d}\right),
\end{aligned}
$$

where $\eta_{\omega_{E}}(y):=\left(\eta_{T_{+}}^{2}(y)+\eta_{T_{-}}^{2}(y)\right)^{1 / 2}$ and $\eta_{\omega_{E}}(\bar{p}), o s c_{\omega_{E}}\left(y^{d}\right)$ are defined analogously.

Proof. We denote by $\varphi_{E}=\prod_{i=1}^{2} \lambda_{i}(E)$, the edge bubble function associated with $E \in \mathcal{E}_{\ell}(\Omega)$ (cf., e.g., [41]). We set $\zeta_{E}:=\left.\left(\nu_{E} \cdot\left[\nabla y_{\ell}\right]\right)\right|_{E}$ and $\xi_{\ell}:=\tilde{\zeta}_{E} \varphi_{E}$, where $\tilde{\zeta}_{E}$ is the extension of $\zeta_{E}$ to $\omega_{E}$ as in [41]. Taking 
advantage of the fact that $\xi_{\ell}$ is an admissible test function in (2.6a) and $\left.\Delta y_{\ell}\right|_{T}=0$, it follows that

$$
\begin{aligned}
\eta_{E}^{2}(y) \lesssim & h_{E}\left(\nu_{E} \cdot\left[\nabla y_{\ell}\right], \zeta_{E} \varphi_{E}\right)_{0, E}= \\
= & h_{E} \sum_{T \subset \omega_{E}}\left(\left(\nu_{\partial T} \cdot \nabla y_{\ell}, \xi_{\ell}\right)_{0, \partial T}-\left(\Delta y_{\ell}, \xi_{\ell}\right)_{0, T}\right)= \\
= & h_{E}\left(\left(\nabla\left(y_{\ell}-y\right), \nabla \xi_{\ell}\right)_{0, \omega_{E}}+\left(c\left(y_{\ell}-y\right), \xi_{\ell}\right)_{0, \omega_{E}}+\right. \\
& \left.\left(u-u_{\ell}, \xi_{\ell}\right)_{0, \omega_{E}}+\left(u_{\ell}-c y_{\ell}, \xi_{\ell}\right)_{0, \omega_{E}}\right) .
\end{aligned}
$$

Standard estimates for $\xi_{\ell}$ (cf., e.g., [41]) readily give (6.4). The estimate (6.5) can be proved along the same lines.

Proof of Theorem 6.1. The efficiency estimate (6.1) follows by summing up the estimates (6.3)-(6.5) over all $T \in \mathcal{T}_{\ell}(\Omega)$ and $E \in \mathcal{E}_{\ell}(\Omega)$. Using the fact that the union of the patches $\omega_{E}$ has a finite overlap allows to conclude.

\section{Numerical Results}

In this section, we illustrate the approximation of state constrained optimal control problems by its Lavrentiev type regularizations using two numerical examples representing two different instances of the possible structure of the Lagrange multiplier according to the classification by Bergounioux and Kunisch [8]. The first example features a solution $y$ that strongly oscillates around the origin where the coincidence set is a connected subdomain with smooth boundary. In contrast to that, the second example, which is taken from [32], features a multiplier in $\mathcal{M}_{+}(\Omega)$ where the coincidence set degenerates to a single point. In both cases the adaptive process generates finite element meshes that are close to those created when one uses the adaptive strategy for state constrained problems as suggested in [26].

\section{Example 1 (Simply connected coincidence set with smooth} boundary): The data of the problem are as follows

$$
\begin{aligned}
\Omega & :=(-2,2)^{2}, \quad \psi:=0 \quad, \quad \alpha:=0.1 \quad, \quad c=0 \quad, \quad \Gamma_{D}:=\partial \Omega, \\
y^{d} & :=y(r)+\Delta p(r)+\sigma(r) \quad, \quad u^{d}:=u(r)+\alpha^{-1} p(r) .
\end{aligned}
$$

Here, $y=y(r), u=u(r), p=p(r)$ and $\sigma=\sigma(r), r:=\left(x_{1}^{2}+x_{2}^{2}\right)^{1 / 2}$, $\left(x_{1}, x_{2}\right)^{T} \in \Omega$, represent the exact optimal solution of the pure state 
constrained problem $(\varepsilon=0)$ according to

$$
\begin{aligned}
y(r):=-r^{\frac{4}{3}} \gamma_{1}(r) & \quad u(r):=-\Delta y(r), \\
p(r):=\gamma_{2}(r)\left(r^{4}-\frac{3}{2} r^{3}+\frac{9}{16} r^{2}\right) & , \quad \sigma(r):=\left\{\begin{array}{c}
0, r<0.75 \\
0.1, \text { otherwise }
\end{array},\right.
\end{aligned}
$$

where

$$
\begin{aligned}
& \gamma_{1}:=\left\{\begin{array}{ll}
1, & r<0.25 \\
-192(r-0.25)^{5}+240(r-0.25)^{4}- & \\
-80(r-0.25)^{3}+1, & 0.25<r<0.75 \\
0, & \text { otherwise }
\end{array},\right. \\
& \gamma_{2}:=\left\{\begin{array}{l}
1, r<0.75 \\
0, \text { otherwise }
\end{array} .\right.
\end{aligned}
$$

Figure 1 shows the computed optimal state $y_{\ell}$ and optimal control $u_{\ell}$ in case of an adaptively generated simplicial triangulation with 9194 degrees of freedom and $\epsilon=10^{-6}$, whereas Figure 2 displays the adaptively generated meshes after 12 (left) and 14 (right) refinement steps for $\epsilon=10^{-6}$.
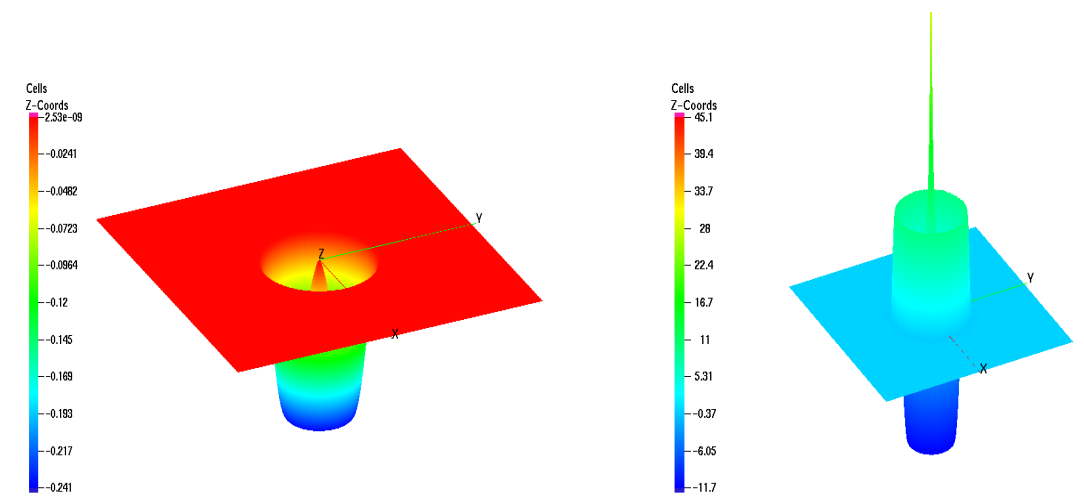

Figure 1. Example 1: Visualization of the discrete optimal state $y_{l}$ (left) and the discrete optimal control $u_{l}$ (right) on a triangulation with 9194 nodes and with regularization parameter $\epsilon=10^{-6}$

Table 1 documents the convergence history of the adaptive refinement process with respect to the convergence of the solutions of the discrete mixed control-state problems $\left(\varepsilon=10^{-6}\right)$ to the exact solution of the pure state constrained problem. We remark that the impact of the regularization parameter $\varepsilon$ has to be observed in the error estimates. In particular, Table 1 contains the $H^{1}$-error in the state, the $L^{2}$-errors in the control and in the adjoint state as well as the $H^{1}$-error 

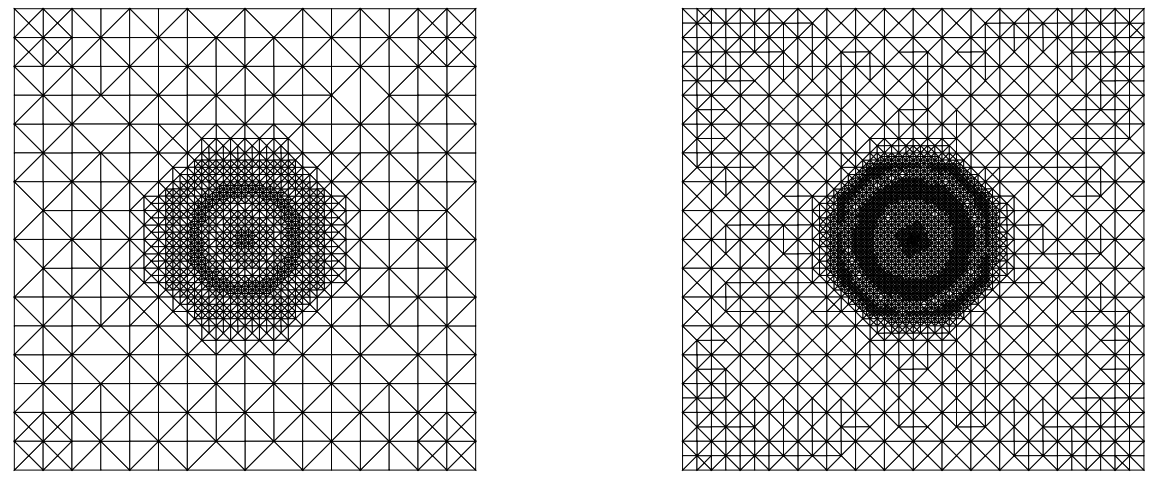

Figure 2. Example 1: Adaptively generated grid after 12 (left) and 14 (right) refinement steps, $\Theta_{i}=0.7, \epsilon=$ $10^{-6}$

TABlE 1. Example 1: Convergence history of the adaptive FEM. Errors in the state, the control, the adjoint state, and the regularized adjoint state $\left(\varepsilon=10^{-6}\right)$

\begin{tabular}{|r|r||c|c|c|c|}
\hline$\ell$ & $N_{\text {dof }}$ & $\left\|u-u_{\ell}\right\|_{0}$ & $\left\|y-y_{\ell}\right\|_{1}$ & $\left\|\bar{p}-\bar{p}_{\ell}\right\|_{1}$ & $\left\|p-p_{\ell}\right\|_{0}$ \\
\hline 1 & 13 & $1.37 \mathrm{e}+01$ & $1.03 \mathrm{e}+00$ & $1.91 \mathrm{e}-01$ & $9.67 \mathrm{e}-01$ \\
2 & 41 & $1.01 \mathrm{e}+01$ & $1.58 \mathrm{e}+00$ & $1.51 \mathrm{e}-01$ & $8.61 \mathrm{e}-01$ \\
4 & 74 & $9.53 \mathrm{e}+00$ & $1.31 \mathrm{e}+00$ & $1.01 \mathrm{e}-01$ & $9.48 \mathrm{e}-02$ \\
6 & 142 & $6.01 \mathrm{e}+00$ & $6.56 \mathrm{e}-01$ & $1.12 \mathrm{e}-01$ & $3.06 \mathrm{e}-02$ \\
8 & 290 & $3.36 \mathrm{e}+00$ & $3.72 \mathrm{e}-01$ & $1.27 \mathrm{e}-01$ & $1.94 \mathrm{e}-02$ \\
10 & 623 & $2.19 \mathrm{e}+00$ & $2.34 \mathrm{e}-01$ & $1.29 \mathrm{e}-01$ & $8.47 \mathrm{e}-03$ \\
12 & 1412 & $1.47 \mathrm{e}+00$ & $1.32 \mathrm{e}-01$ & $1.31 \mathrm{e}-01$ & $1.08 \mathrm{e}-02$ \\
14 & 3498 & $1.01 \mathrm{e}+00$ & $7.92 \mathrm{e}-02$ & $1.30 \mathrm{e}-01$ & $1.29 \mathrm{e}-02$ \\
\hline
\end{tabular}

TABLE 2. Example 1: Convergence history of the adaptive FEM. Discretization errors in the pure state constrained case $(\varepsilon=0)$; from $[26]$

\begin{tabular}{|r|r||c|c|c|c|}
\hline$\ell$ & $N_{\text {dof }}$ & $\left\|u-u_{\ell}\right\|_{0}$ & $\left\|y-y_{\ell}\right\|_{1}$ & $\left\|\bar{p}-\bar{p}_{\ell}\right\|_{1}$ & $\left\|p-p_{\ell}\right\|_{0}$ \\
\hline 1 & 13 & $2.37 \mathrm{e}+01$ & $1.51 \mathrm{e}+00$ & $6.74 \mathrm{e}-01$ & $2.06 \mathrm{e}+00$ \\
2 & 41 & $1.35 \mathrm{e}+01$ & $1.02 \mathrm{e}+00$ & $1.06 \mathrm{e}-01$ & $1.28 \mathrm{e}-01$ \\
4 & 105 & $9.41 \mathrm{e}+00$ & $7.34 \mathrm{e}-01$ & $7.88 \mathrm{e}-02$ & $9.54 \mathrm{e}-02$ \\
6 & 244 & $6.01 \mathrm{e}+00$ & $5.41 \mathrm{e}-01$ & $6.02 \mathrm{e}-02$ & $4.78 \mathrm{e}-02$ \\
8 & 532 & $3.18 \mathrm{e}+00$ & $2.80 \mathrm{e}-01$ & $4.53 \mathrm{e}-02$ & $3.92 \mathrm{e}-02$ \\
10 & 1147 & $1.91 \mathrm{e}+00$ & $1.74 \mathrm{e}-01$ & $3.44 \mathrm{e}-02$ & $2.36 \mathrm{e}-02$ \\
12 & 2651 & $1.29 \mathrm{e}+00$ & $1.03 \mathrm{e}-01$ & $2.02 \mathrm{e}-02$ & $1.81 \mathrm{e}-02$ \\
14 & 6340 & $9.74 \mathrm{e}-01$ & $6.32 \mathrm{e}-02$ & $1.17 \mathrm{e}-02$ & $1.22 \mathrm{e}-02$ \\
\hline
\end{tabular}

in the regularized adjoint state. The adaptive refinement process has been terminated when the size of the regularization parameter $\varepsilon=10^{-6}$ 
started to blur the results. The same effect could be observed for larger values of $\varepsilon$ (see also Figure 3 (right)). For a comparison, Table 2 shows the decrease of the discretization errors in the limiting case $\varepsilon=0$ (pure state constraints). We observe that in this example the most significant impact of the regularization parameter is on the errors in the adjoint state and the regularized adjoint state.

Figure 3 (left) illustrates the benefit of adaptive versus uniform refinement by showing on a logarithmic scale the error in the control as a function of the degrees of freedom $\left(\epsilon=10^{-6}\right)$. Figure 3 (right) contains a comparison of the error in the control for the pure state constrained problem $(\epsilon=0)$ and its Lavrentiev regularizations $(\varepsilon=$ $\left.10^{-2}, 10^{-4}, 10^{-6}\right)$.
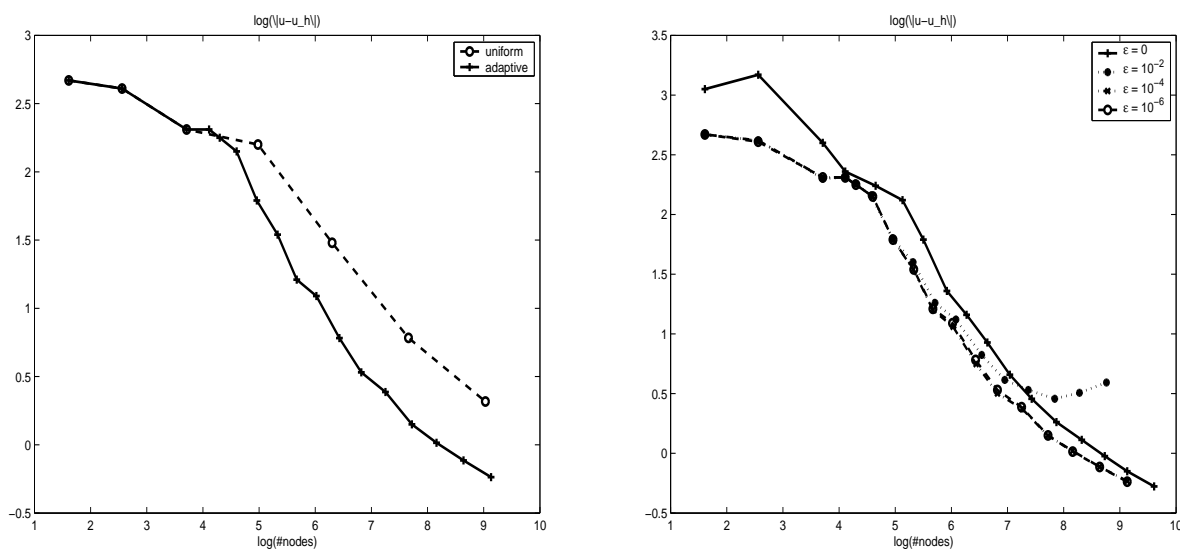

FiguRE 3. Example 1: Adaptive refinement [straight line] versus uniform refinement [dotted line] (left) and discretization error in the control for different regularization parameters (right)

The residual-type estimators $\eta_{\ell}(y)$ in the state and $\eta_{\ell}(\bar{p})$ in the regularized adjoint state as well as the data oscillations $\operatorname{osc}_{\ell}\left(u^{d}\right)$ in the shift control and ${ } s c_{\ell}\left(y^{d}\right)$ in the desired state are given in Table 3. Again, for a comparison with the pure state constrained case, Table 4 contains the corresponding values in case $\varepsilon=0$. 
TABLE 3. Example 1: Convergence history of the adaptive FEM. Estimator in the state and regularized adjoint state, data oscillations $\left(\varepsilon=10^{-6}\right)$

\begin{tabular}{|r|r||c|c|c|c|}
\hline$\ell$ & $N_{\text {dof }}$ & $\eta_{\ell}(y)$ & $\eta_{\ell}(\bar{p})$ & osc $_{\ell}\left(u^{d}\right)$ & $o s c_{\ell}\left(y^{d}\right)$ \\
\hline 1 & 13 & $4.20 \mathrm{e}+00$ & $1.04 \mathrm{e}+00$ & $1.37 \mathrm{e}+01$ & $5.42 \mathrm{e}-01$ \\
2 & 41 & $4.25 \mathrm{e}+00$ & $1.04 \mathrm{e}+00$ & $1.36 \mathrm{e}+01$ & $6.22 \mathrm{e}-01$ \\
4 & 74 & $4.01 \mathrm{e}+00$ & $4.71 \mathrm{e}-01$ & $9.67 \mathrm{e}+00$ & $3.32 \mathrm{e}-01$ \\
6 & 142 & $1.77 \mathrm{e}+00$ & $3.14 \mathrm{e}-01$ & $6.03 \mathrm{e}+00$ & $1.11 \mathrm{e}-01$ \\
8 & 290 & $1.27 \mathrm{e}+00$ & $2.49 \mathrm{e}-01$ & $3.38 \mathrm{e}+00$ & $5.36 \mathrm{e}-02$ \\
10 & 623 & $8.70 \mathrm{e}-01$ & $1.80 \mathrm{e}-01$ & $2.19 \mathrm{e}+00$ & $2.78 \mathrm{e}-02$ \\
12 & 1412 & $5.50 \mathrm{e}-01$ & $1.12 \mathrm{e}-01$ & $1.47 \mathrm{e}+00$ & $1.50 \mathrm{e}-02$ \\
14 & 3498 & $3.42 \mathrm{e}-01$ & $6.90 \mathrm{e}-02$ & $1.01 \mathrm{e}+00$ & $7.08 \mathrm{e}-03$ \\
\hline
\end{tabular}

TABle 4. Example 1: Convergence history of the adaptive FEM. Estimators and data oscillations in the pure state constrained case $(\varepsilon=0)$; from $[26]$

\begin{tabular}{|r|r||c|c|c|c|}
\hline$\ell$ & $N_{\text {dof }}$ & $\eta_{\ell}(y)$ & $\eta_{\ell}(\bar{p})$ & osc $_{\ell}\left(u^{d}\right)$ & osc $_{\ell}\left(y^{d}\right)$ \\
\hline 1 & 13 & $2.19 \mathrm{e}+01$ & $2.04 \mathrm{e}+00$ & $1.37 \mathrm{e}+01$ & $5.42 \mathrm{e}-01$ \\
2 & 41 & $9.83 \mathrm{e}+00$ & $8.10 \mathrm{e}-01$ & $1.36 \mathrm{e}+01$ & $6.22 \mathrm{e}-01$ \\
4 & 105 & $3.67 \mathrm{e}+00$ & $4.35 \mathrm{e}-01$ & $9.42 \mathrm{e}+00$ & $3.32 \mathrm{e}-01$ \\
6 & 244 & $1.63 \mathrm{e}+00$ & $2.60 \mathrm{e}-01$ & $5.99 \mathrm{e}+00$ & $1.11 \mathrm{e}-01$ \\
8 & 532 & $1.17 \mathrm{e}+00$ & $1.69 \mathrm{e}-01$ & $3.17 \mathrm{e}+00$ & $4.47 \mathrm{e}-02$ \\
10 & 1147 & $7.72 \mathrm{e}-01$ & $1.22 \mathrm{e}-01$ & $1.90 \mathrm{e}+00$ & $2.17 \mathrm{e}-02$ \\
12 & 2651 & $4.71 \mathrm{e}-01$ & $7.37 \mathrm{e}-02$ & $1.29 \mathrm{e}+00$ & $9.27 \mathrm{e}-03$ \\
14 & 6340 & $2.93 \mathrm{e}-01$ & $4.55 \mathrm{e}-02$ & $9.74 \mathrm{e}-01$ & $4.62 \mathrm{e}-03$ \\
\hline
\end{tabular}

Example 2 (Degenerated coincidence set [32]): The data of the problem are as follows

$$
\begin{aligned}
\Omega & :=B(0,1), \Gamma_{D}=\emptyset, \quad \alpha:=1.0 \quad, \quad c=1.0, \\
y^{d}(r) & :=4+\frac{1}{\pi}-\frac{1}{4 \pi} r^{2}+\frac{1}{2 \pi} \ln (r), \\
u^{d}(r) & :=4+\frac{1}{4 \pi} r^{2}-\frac{1}{2 \pi} \ln (r) \quad, \quad \psi(r):=r+4 .
\end{aligned}
$$

The optimal solution in the pure state constrained case is given by:

$$
\begin{array}{ll}
y(r) \equiv 4 & , \quad p(r)=\frac{1}{4 \pi} r^{2}-\frac{1}{2 \pi} \ln (r), \\
u(r) \equiv 4 & , \quad \sigma=\delta_{0} .
\end{array}
$$

Figure 4 displays the computed optimal state $y_{\ell}$ and optimal control $u_{\ell}$ for a simplicial triangulation with 964 degrees of freedom. For the regularization parameter $\epsilon=10^{-6}$, the adaptively generated grids after 12 and 14 refinement steps are shown in Figure 5. 

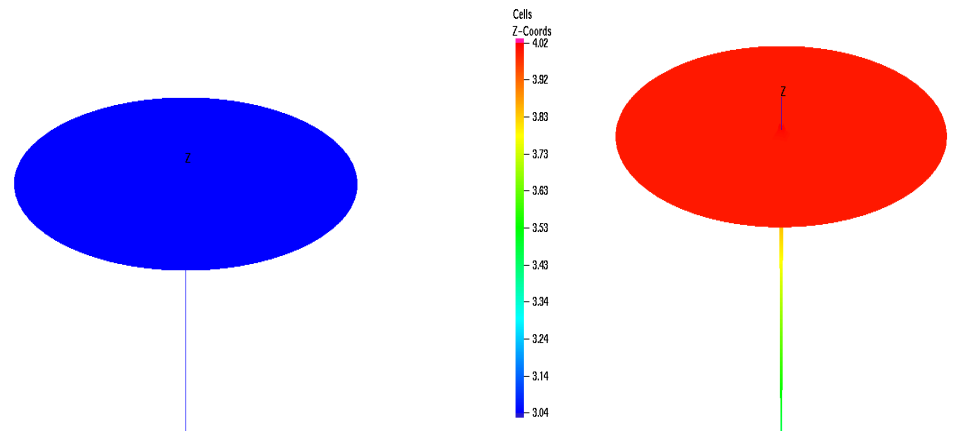

Figure 4. Example 2: Visualization of the discrete state $y_{l}$ (left) and the discrete control $u_{l}$ (right) on an adaptive generated mesh with 964 nodes and with regularization parameter $\epsilon=10^{-6}$
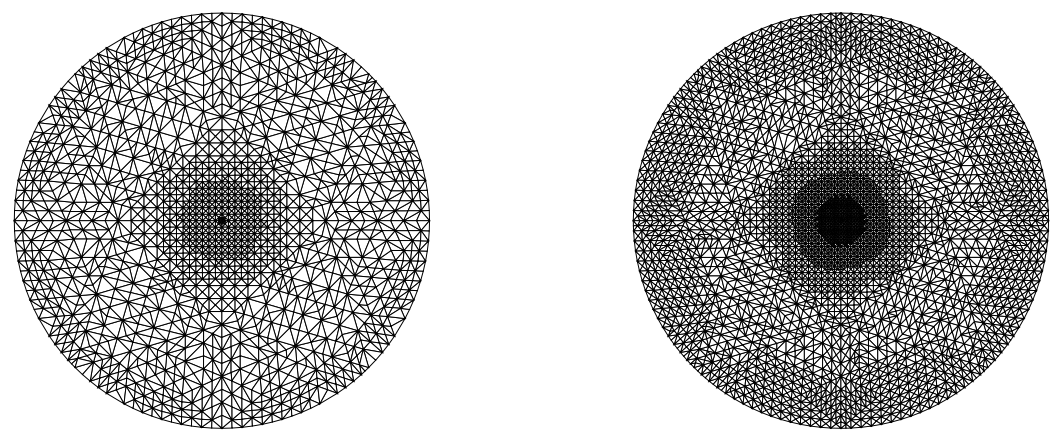

Figure 5. Example 2: Adaptively generated grid after 12 (left) and 14 (right) refinement steps, $\Theta_{i}=0.7, \epsilon=$ $10^{-6}$

In case $\varepsilon=10^{-6}$, Table 5 and Table 7 reflect the convergence history of the adaptive refinement process with data analogous to those in Example 1. As before, in order to compare with the pure state constrained case $(\varepsilon=0)$, the associated data are given in Table 6 and Table 8 . We remark that there are no data oscillations in $\psi$ in the pure state constrained case $\varepsilon=0$, since $\left\langle\sigma-\sigma_{\ell}, \psi-\psi_{\ell}\right\rangle=0$ where $\langle\cdot, \cdot\rangle$ stands for the dual pairing between the space of Radon measures and the space of continuous functions (cf. [26]). We see that here the impact of the regularization parameter is less pronounced than in the first example (provided $\varepsilon$ is chosen sufficiently small; see also Figure 6 (right)). The benefit of adaptive versus uniform refinement is addressed in Figure 6 (left) which displays on a logarithmic scale the discretization error in 
TABle 5. Example 2: Convergence history of the adaptive FEM. Errors in the state, the control, the adjoint state, and the regularized adjoint state $\left(\varepsilon=10^{-6}\right)$

\begin{tabular}{|r|r||c|c|c|c|}
\hline$\ell$ & $N_{\text {dof }}$ & $\left\|u-u_{\ell}\right\|_{0}$ & $\left\|y-y_{\ell}\right\|_{1}$ & $\left\|\bar{p}-\bar{p}_{\ell}\right\|_{1}$ & $\left\|p-p_{\ell}\right\|_{0}$ \\
\hline 1 & 13 & $8.62 \mathrm{e}-02$ & $1.75 \mathrm{e}-02$ & $2.24 \mathrm{e}-02$ & $6.73 \mathrm{e}-02$ \\
2 & 41 & $6.50 \mathrm{e}-02$ & $1.01 \mathrm{e}-02$ & $1.21 \mathrm{e}-02$ & $2.93 \mathrm{e}-02$ \\
4 & 73 & $5.56 \mathrm{e}-02$ & $6.89 \mathrm{e}-03$ & $9.36 \mathrm{e}-03$ & $1.54 \mathrm{e}-02$ \\
6 & 121 & $3.39 \mathrm{e}-02$ & $2.34 \mathrm{e}-03$ & $6.02 \mathrm{e}-03$ & $8.30 \mathrm{e}-03$ \\
8 & 243 & $1.98 \mathrm{e}-02$ & $6.96 \mathrm{e}-04$ & $3.91 \mathrm{e}-03$ & $4.35 \mathrm{e}-03$ \\
10 & 603 & $1.14 \mathrm{e}-02$ & $2.25 \mathrm{e}-04$ & $2.32 \mathrm{e}-03$ & $2.00 \mathrm{e}-03$ \\
12 & 1618 & $6.39 \mathrm{e}-03$ & $6.98 \mathrm{e}-05$ & $1.46 \mathrm{e}-03$ & $9.37 \mathrm{e}-04$ \\
14 & 3989 & $3.55 \mathrm{e}-03$ & $2.58 \mathrm{e}-05$ & $8.54 \mathrm{e}-04$ & $4.57 \mathrm{e}-04$ \\
16 & 10656 & $1.95 \mathrm{e}-03$ & $1.10 \mathrm{e}-05$ & $4.76 \mathrm{e}-04$ & $2.21 \mathrm{e}-04$ \\
\hline
\end{tabular}

TABLE 6. Example 2: Convergence history of the adaptive FEM. Discretization errors in the pure state constrained case $(\varepsilon=0)$; from $[26]$

\begin{tabular}{|r|r||c|c|c|c|}
\hline$\ell$ & $N_{\text {dof }}$ & $\left\|u-u_{\ell}\right\|_{0}$ & $\left\|y-y_{\ell}\right\|_{1}$ & $\left\|\bar{p}-\bar{p}_{\ell}\right\|_{1}$ & $\left\|p-p_{\ell}\right\|_{0}$ \\
\hline 1 & 13 & $1.04 \mathrm{e}-01$ & $8.51 \mathrm{e}-03$ & $1.74 \mathrm{e}-02$ & $3.73 \mathrm{e}-02$ \\
2 & 41 & $6.95 \mathrm{e}-02$ & $4.43 \mathrm{e}-03$ & $9.01 \mathrm{e}-03$ & $1.86 \mathrm{e}-02$ \\
4 & 73 & $5.73 \mathrm{e}-02$ & $2.30 \mathrm{e}-03$ & $7.36 \mathrm{e}-03$ & $1.00 \mathrm{e}-02$ \\
6 & 121 & $3.42 \mathrm{e}-02$ & $1.79 \mathrm{e}-03$ & $6.11 \mathrm{e}-03$ & $7.41 \mathrm{e}-03$ \\
8 & 243 & $1.99 \mathrm{e}-02$ & $1.07 \mathrm{e}-03$ & $4.02 \mathrm{e}-03$ & $4.13 \mathrm{e}-03$ \\
10 & 604 & $1.14 \mathrm{e}-02$ & $4.02 \mathrm{e}-04$ & $2.43 \mathrm{e}-03$ & $1.95 \mathrm{e}-03$ \\
12 & 1621 & $6.39 \mathrm{e}-03$ & $1.60 \mathrm{e}-04$ & $1.52 \mathrm{e}-03$ & $9.26 \mathrm{e}-04$ \\
14 & 3991 & $3.55 \mathrm{e}-03$ & $6.81 \mathrm{e}-05$ & $8.79 \mathrm{e}-04$ & $4.55 \mathrm{e}-04$ \\
\hline
\end{tabular}

TABLE 7. Example 2: Convergence history of the adaptive FEM. Estimators and data oscillations $\left(\varepsilon=10^{-6}\right)$

\begin{tabular}{|r|r||c|c|c|c|c|}
\hline$\ell$ & $N_{\text {dof }}$ & $\eta_{\ell}(y)$ & $\eta_{\ell}(\bar{p})$ & osc $_{\ell}\left(u^{d}\right)$ & osc $_{\ell}(\psi)$ & osc $_{\ell}\left(y^{d}\right)$ \\
\hline 1 & 13 & $6.15 \mathrm{e}-02$ & $7.38 \mathrm{e}-02$ & $1.29 \mathrm{e}-01$ & $1.11 \mathrm{e}-01$ & $4.36 \mathrm{e}-02$ \\
2 & 41 & $2.29 \mathrm{e}-02$ & $3.76 \mathrm{e}-02$ & $8.14 \mathrm{e}-02$ & $3.25 \mathrm{e}-02$ & $1.26 \mathrm{e}-02$ \\
4 & 73 & $1.00 \mathrm{e}-02$ & $2.52 \mathrm{e}-02$ & $5.95 \mathrm{e}-02$ & $2.13 \mathrm{e}-02$ & $7.78 \mathrm{e}-03$ \\
6 & 121 & $3.11 \mathrm{e}-03$ & $2.01 \mathrm{e}-02$ & $3.56 \mathrm{e}-02$ & $1.23 \mathrm{e}-02$ & $4.96 \mathrm{e}-03$ \\
8 & 243 & $9.15 \mathrm{e}-04$ & $1.32 \mathrm{e}-02$ & $2.06 \mathrm{e}-02$ & $5.27 \mathrm{e}-03$ & $1.87 \mathrm{e}-03$ \\
10 & 603 & $2.59 \mathrm{e}-04$ & $8.12 \mathrm{e}-03$ & $1.17 \mathrm{e}-02$ & $2.25 \mathrm{e}-03$ & $8.28 \mathrm{e}-04$ \\
12 & 1618 & $7.23 \mathrm{e}-05$ & $4.76 \mathrm{e}-03$ & $6.54 \mathrm{e}-03$ & $9.86 \mathrm{e}-04$ & $3.17 \mathrm{e}-04$ \\
14 & 3989 & $2.01 \mathrm{e}-05$ & $2.89 \mathrm{e}-03$ & $3.62 \mathrm{e}-03$ & $3.95 \mathrm{e}-04$ & $1.42 \mathrm{e}-04$ \\
16 & 10656 & $5.53 \mathrm{e}-06$ & $1.78 \mathrm{e}-03$ & $1.98 \mathrm{e}-03$ & $1.86 \mathrm{e}-04$ & $5.89 \mathrm{e}-05$ \\
\hline
\end{tabular}

the control as a function of the degrees of freedom (dotted line: adaptive refinement, straight line: uniform refinement, $\varepsilon=10^{-6}$ ). Finally, Figure 6 (right) contains a comparison of the error in the control for 
TABle 8. Example 2: Convergence history of the adaptive FEM. Estimators and data oscillations in the pure state constrained case $(\varepsilon=0)$; from [26]

\begin{tabular}{|r|r||c|c|c|c|}
\hline$\ell$ & $N_{\text {dof }}$ & $\eta_{\ell}(y)$ & $\eta_{\ell}(\bar{p})$ & $o s c_{\ell}\left(u^{d}\right)$ & osc $_{\ell}\left(y^{d}\right)$ \\
\hline 1 & 13 & $7.32 \mathrm{e}-02$ & $7.62 \mathrm{e}-02$ & $1.29 \mathrm{e}-01$ & $4.36 \mathrm{e}-02$ \\
2 & 41 & $2.45 \mathrm{e}-02$ & $3.83 \mathrm{e}-02$ & $8.14 \mathrm{e}-02$ & $1.26 \mathrm{e}-02$ \\
4 & 73 & $1.02 \mathrm{e}-02$ & $2.54 \mathrm{e}-02$ & $5.95 \mathrm{e}-02$ & $7.78 \mathrm{e}-03$ \\
6 & 121 & $3.11 \mathrm{e}-03$ & $1.97 \mathrm{e}-02$ & $3.56 \mathrm{e}-02$ & $4.96 \mathrm{e}-03$ \\
8 & 243 & $9.10 \mathrm{e}-04$ & $1.32 \mathrm{e}-02$ & $2.06 \mathrm{e}-02$ & $1.87 \mathrm{e}-03$ \\
10 & 604 & $2.59 \mathrm{e}-04$ & $8.07 \mathrm{e}-03$ & $1.17 \mathrm{e}-02$ & $8.27 \mathrm{e}-04$ \\
12 & 1621 & $7.22 \mathrm{e}-05$ & $4.75 \mathrm{e}-03$ & $6.54 \mathrm{e}-03$ & $3.16 \mathrm{e}-04$ \\
14 & 3991 & $2.01 \mathrm{e}-05$ & $2.89 \mathrm{e}-03$ & $3.62 \mathrm{e}-03$ & $1.41 \mathrm{e}-04$ \\
\hline
\end{tabular}
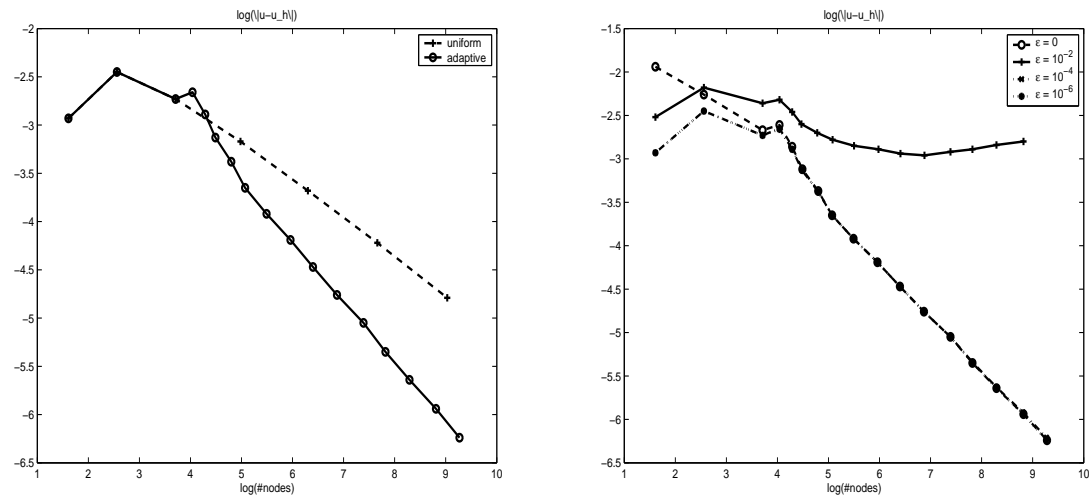

Figure 6. Example 2: Adaptive versus uniform refinement (left) and discretization error in the control for different regularization parameters (right)

the pure state constrained problem $(\epsilon=0)$ and its Lavrentiev regularizations $\left(\varepsilon=10^{-2}, 10^{-4}, 10^{-6}\right)$.

Acknowledgments. The authors acknowledge support by the NSF under Grant No. DMS-0411403 and Grant No. DMS-0511611 as well as by the DFG within the Priority Program SPP 1253 'PDE Constrained Optimization'.

\section{REFERENCES}

[1] M. Ainsworth and J.T. Oden (2000). A Posteriori Error Estimation in Finite Element Analysis. Wiley, Chichester.

[2] N. ARAda AND J.P. RAYMOnd (2000). Optimal control problems with mixed control-state constraints. SIAM J. Control Optim. 39, 1391-1407.

[3] I. Babuska And T. Strouboulis (2001). The Finite Element Method and its Reliability. Clarendon Press, Oxford. 
[4] W. Bangerth and R. Rannacher (2003). Adaptive Finite Element Methods for Differential Equations. Lectures in Mathematics. ETH-Zürich. Birkhäuser, Basel.

[5] R. Becker, H. Kapp, and R. Rannacher (2000). Adaptive finite element methods for optimal control of partial differential equations: Basic concepts. SIAM J. Control Optim. 39, 113-132.

[6] M. Bergounioux, K. Ito, And K. Kunisch (1999). Primal-dual strategy for constrained optimal control problems. SIAM J. Control and Optimization 34, 1176-1194

[7] M. Bergounioux, M. Haddou, M. Hintermüller, and K. Kunisch (2000). A comparison of a Moreau-Yosida based active set strategy and interior point methods for constrained optimal control problems. SIAM J. Optim. 11, 495-521.

[8] M. Bergounioux And K. Kunisch (2002). On the structure of the Lagrange multiplier for state-constrained optimal control problems. Systms and Control Letetrs 48, 169-176.

[9] E. Casas (1986). Control of an elliptic problem with pointwise state controls. SIAM J. Control Optim. 24, 1309-1318.

[10] E. Casas And M. Mateos (2002). Uniform convergence of the FEM applications to state constrained control problems. Comp. Appl. Math. 21, 67-100.

[11] E. Casas, J.-P. RAYMOND, AND H. Zidani (2000). Pontryagin's principle for local solutions of control problems with mixed control-state constraints. SIAM J. Control Optim. 39, 1182-1203.

[12] K. Deckelnick And M. Hinze (2006). Convergence of a finite element approximation to a state constrained elliptic control problem. Preprint, Dept. of Mathematics, Dresden University of Technology.

[13] W. DöRfler (1996). A convergent adaptive algorithm for Poisson's equation. SIAM J. Numer. Anal. 33, 1106-1124.

[14] K. Eriksson, D. Estep, P. Hansbo, And C. Johnson (1995). Computational Differential Equations. Cambridge University Press, Cambridge.

[15] F. FALK (1973). Approximation of a class of optimal control problems with order of convergence estimates. J. Math. Anal. Appl. 44, 28-47.

[16] A. Gaevskaya, R.H.W. Hoppe, Y. Iliash, and M. Kieweg (2006). A posteriori error analysis of control constrained distributed and boundary control problems. Proc. Conf. Advances in Scientific Computing, Moscow, Russia (O. Pironneau et al.; eds.), Russian Academy of Sciences, Moscow.

[17] A. Gaevskaya, R.H.W. Hoppe, Y. Iliash, and M. Kieweg (2007). Convergence analysis of an adaptive finite element method for distributed control problems with control constraints. Proc. Conf. Optimal Control for PDEs, Oberwolfach, Germany (G. Leugering et al.; eds.), Birkhäuser, Basel.

[18] A. Gaevskaya, R.H.W. Hoppe, and S. Repin (2006). A Posteriori Estimates for Cost Functionals of Optimal Control Problems. In: Numerical Mathematics and Advanced Applications (A. Bermudez de Castro et al.; eds.), pp. 308-316, Springer, Berlin-Heidelberg-New York.

[19] P. Grisvard (1985). Elliptic Problems in Nonsmooth Domains. Pitman, Boston. 
[20] M. Hintermüller And R.H.W. HopPe (2007). Goal-oriented adaptivity in control constrained optimal control of partial differential equations. Submitted to SICON.

[21] M. Hintermüller, R.H.W. Hoppe, Y. Iliash, And M. Kieweg (2007). An a posteriori error analysis of adaptive finite element methods for distributed elliptic control problems with control constraints. to appear in ESAIM, COCV.

[22] M. Hintermüller AND K. Kunisch (2006). Feasible and non-interior pathfollowing in constrained minimization with low multiplier regularity. SIAM J. Control Optim., 45, 1198-1221.

[23] M. Hintermüller And K. Kunisch (2006). Stationary state constrained optimal control problems. Preprint IFB-Report No. 3, Karl-Franzens-University of Graz.

[24] M. Hintermüller And K. Kunisch (2007). Path-following methods for a class of constrained minimization problems in function space. to appear in SIAM J. Optimization.

[25] R.H.W. Hoppe, Y. Iliash, C. Iyyunni, and N. Sweilam (2006). A posteriori error estimates for adaptive finite element discretizations of boundary control problems. J. Numer. Anal. 14, 57-82.

[26] R.H.W. Hoppe AND M. KIEwEG (2007), A posteriori error estimation of finite element approximations of pointwise state constrained distributed control problems. submitted.

[27] K. Kunisch And A. Rösch (2002). Primal-dual active set strategy for a general class of constrained optimal control problems. SIAM J. Control and Optimization 42, 321-334.

[28] R. Li, W. LiU, H. MA, And T. TANG (2002). Adaptive finite element approximation for distributed elliptic optimal control problems. SIAM J. Control Optim. 41, 1321-1349.

[29] J.L. Lions (1971). Optimal Control of Systems Governed by Partial Differential Equations. Springer, Berlin-Heidelberg-New York.

[30] W. LiU AND N. YAN (2001). A posteriori error estimates for distributed optimal control problems. Adv. Comp. Math. 15, 285-309.

[31] W. LiU And N. YAn (2003). A posteriori error estimates for convex boundary control problems. Preprint, Institute of Mathematics and Statistics, University of Kent, Canterbury.

[32] C. Meyer, U. Prüfert, And F. Tröltzsch (2005). On two numerical methods for state-constrained elliptic control problems. Preprint, Dept. of Mathematics, Berlin University of Technology.

[33] C. Meyer, A. Rösch, And F. TröLtzsch (2006). Optimal control problems of PDEs with regularized pointwise state constraints. Comput. Optim. Appl., 33, 209-228.

[34] P. Morin, R.H. Nochetto, and K.G. Siebert (2000). Data oscillation and convergence of adaptive FEM. SIAM J. Numer. Anal., 38, 2, 466-488.

[35] P. NeittaAnmäki And S. Repin (2004). Reliable methods for mathematical modelling. Error control and a posteriori estimates. Elsevier, New York.

[36] U. Prüfert, F. Tröltzsch, And M. Weiser (2007) The convergence of an interior point method for an elliptic control problem with mixed control-state constraints. to appear in Comp. Opt. Appl. 
[37] A. Rösch AND F. Tröltzsch (2006) Sufficient second order optimality conditions for an elliptic optimal control problem with pointwise control-state constraints. SIAM J. Optimization, 17, 776-794.

[38] F. TRÖLTZSCH (1979). A minimum principle and a generalized bang-bang principle for a distributed optimal control problem with constraints on the control and the state. ZAMM 59, 737-739.

[39] F. TröLtzsch (2005a). Optimale Steuerung partieller Differentialgleichungen. Vieweg, Wiesbaden, 2005

[40] F. TröLtzSch (2005) Regular Lagrange multipliers for control problems with mixed pointwise control-state constraints. SIAM J. Optim., 15, 616-634.

[41] R. Verfürth (1996). A Review of A Posteriori Estimation and Adaptive Mesh-Refinement Techniques. Wiley-Teubner, New York, Stuttgart.

[42] B. Vexler AND W. Wollner (2007). Adaptive finite element methods for elliptic optimization problems with control constraints. Preprint, RICAM, Linz.

Department of Mathematics, University of Houston, Houston, tX 77204-3008, USA, And Institute of Mathematics, University of AugsBurg, D-86159 Augsburg, Germany

Department of Mathematics, University of Houston, Houston, TX 77204-3008, USA, And Institute of Mathematics, University of AugsBurg, D-86159 Augsburg, Germany 